\title{
Locally Advanced Primary Small Cell Carcinoma of the Breast: A Case Report and Review of Current Evidence
}

\author{
Linsey Atchison $^{a}$ Thomas Hardy ${ }^{b}$ Tara Mancl ${ }^{c}$ Brian Patrick Quaranta ${ }^{d}$ \\ Ankit Madan ${ }^{\mathrm{e}}$ \\ aDepartment of Internal Medicine Residency, SOVAH, Danville, VA, USA; bepartment of \\ Pathology, SOVAH, Danville, VA, USA; 'Department of General Surgery, SOVAH, Danville, \\ VA, USA; dDepartment of Radiation Oncology, Duke, Raleigh, NC, USA; 'Department of \\ Internal Medicine, Medical Oncology, SOVAH, Danville, VA, USA
}

\section{Keywords}

Small-cell breast carcinoma - Rare breast cancer - Neoadjuvant chemotherapy - Outcomes .

Genetic mutations

\begin{abstract}
Small-cell breast carcinoma (SCBC) is a very rare type of aggressive breast cancer constituting less than $1 \%$ of all breast cancers. The WHO classification categorizes this tumor as small-cell neuroendocrine carcinoma, and its prognosis is usually worse as compared to invasive breast cancers. We report a 64-year-old Caucasian female who presented with a large fungating left breast mass. Biopsy of the mass revealed small-cell carcinoma of the breast, negative for all 3 receptors (estrogen, progesterone, and Her2/neu). Imaging studies were negative for distant metastasis. She was subsequently treated after multidisciplinary discussion utilizing neoadjuvant chemotherapy with platinum agents and etoposide, modified radical mastectomy with axillary lymph node dissection, and adjuvant radiation therapy. Since she has triple-negative small-cell breast cancer, she will be followed with active surveillance without hormonal therapy. With less than 100 cases reported and in the absence of evidence-based standard treatment guidelines, we have reviewed various case series reported in the literature. We further discuss various chemotherapy regimens used previously, adverse prognostic factors of breast neuroendocrine tumors, common receptor status, and genetic mutations found in SCBC.
\end{abstract}




\section{Introduction}

Small-cell breast carcinoma (SCBC) is a rare tumor which represents $<1 \%$ of all breast cancers [1]. Extrapulmonary small-cell tumors (EPSCC) occur commonly in gastrointestinal and genitourinary organs but have been known to occur rarely in breast, larynx, pancreas, hypopharynx, salivary glands, skin, nasal cavity, and paranasal sinuses [2]. EPSCC accounts for $2-5 \%$ of all small-cell tumors [3]. The WHO 2019 breast cancer classification (fifth edition) categorizes this tumor as a small-cell neuroendocrine carcinoma [4]. Pure SCBC typically portends a significantly poorer prognosis as compared to invasive ductal carcinoma. EPSCC also show a very similar histological morphology to the more commonly known small-cell lung carcinoma (SCLC) [5]. Due to the difficulty of histologically distinguishing it from SCLC, metastatic disease must first be ruled out with a thorough search for another primary site. Unlike its extensively researched counterpart, SCLC, tested and proven treatment regimens have yet to be established for SCBC.

Most of the evidence on SCBC is drawn from anecdotal case reports and few case series. Shin et al. [6] and Kanat et al. [7] reported outcomes and management of 9 and 7 patients, respectively. Boutrid et al. [8] reported on 56 cases cited in the literature.

Since there is still a limited amount of literature on SCBC due to its rarity, reporting each verified case remains invaluable for future studies on this very rare and aggressive form of breast cancer. We report a 64-year-old female with locally advanced SCBC who was treated utilizing a multidisciplinary approach involving neoadjuvant chemotherapy, modified radical mastectomy with axillary lymph node dissection, and adjuvant radiation therapy (RT) to the axilla.

\section{Case Presentation}

A 64-year-old Caucasian female presented to urgent care with the complaint of a large left breast mass. She first noticed the mass on the outer upper quadrant of the left breast 4 months prior. Due to the COVID-19 pandemic, she did not seek immediate medical attention. Subsequently, over the next few months, the lesion grew into a painful fungating, red mass protruding out of the breast (Fig. 1). Once she noticed significant colorless, foul-smelling discharge from the left breast she decided to seek medical care. She has a 30 pack-year smoking history but quit smoking several years ago. Her family history is significant for lung cancer in 2 of her aunts. There was no other significant medical or surgical history. She was not taking any medications.

Clinical exam revealed an 11-cm left breast mass with serosanguinous drainage. The breast mass was fungating with a 2-cm ulcer on the lateral border and she had palpable left axillary lymphadenopathy. Her lungs were clear to auscultation, heart auscultation revealed normal rate and rhythm. She did not have cervical, inguinal, and right axillary lymphadenopathy. Her neurological exam was normal.

She was referred to a general surgeon and underwent a left breast biopsy. The biopsy revealed a necrotic tumor consistent with small cell carcinoma (Fig. 2). Immunohistochemistry analysis revealed uniform staining with SOX-10, cytokeratin (CK), and variable staining with CK 7, TTF-1 (thyroid transcription factor-1), and GATA 3. Immunohistochemical stains were weakly to moderately positive for CD56, synaptophysin, and neuron specific enolase (NSE). Ki67 was greater than 70\%. Estrogen receptor, progesterone receptor, and Her2/neu stains were all negative.

She was evaluated by medical oncology and to rule out primary SCLC, a PET scan was performed. The PET-CT scan revealed a 15-cm left breast tumor with an SUV of 6.84, along 


\section{Case Reports in Oncology}

\begin{tabular}{l|l}
\hline Case Rep Oncol 2021;14:761-766 \\
\hline DOI: 10.1159/000515505 & $\begin{array}{l}\text { @ 2021 The Author(s). Published by S. Karger AG, Basel } \\
\text { www.karger.com/cro }\end{array}$ \\
\hline
\end{tabular}

Atchison et al.: Locally Advanced Small Cell Carcinoma of the Breast
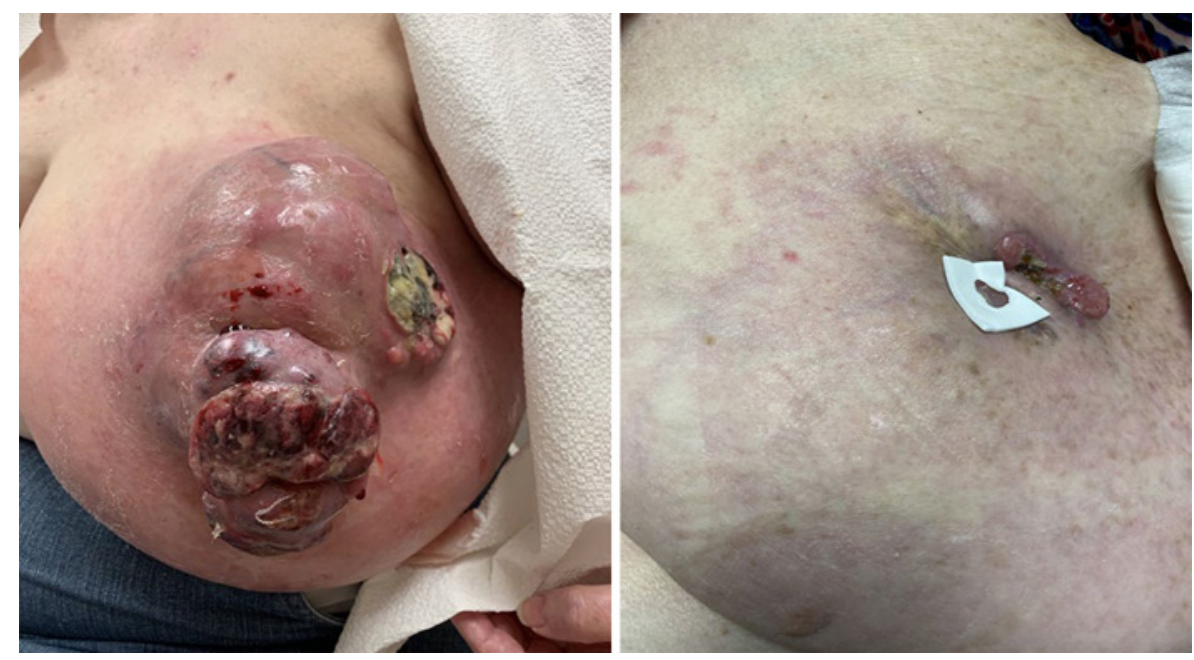

Fig. 1. Breast mass before and after 5 cycles of neoadjuvant chemotherapy.
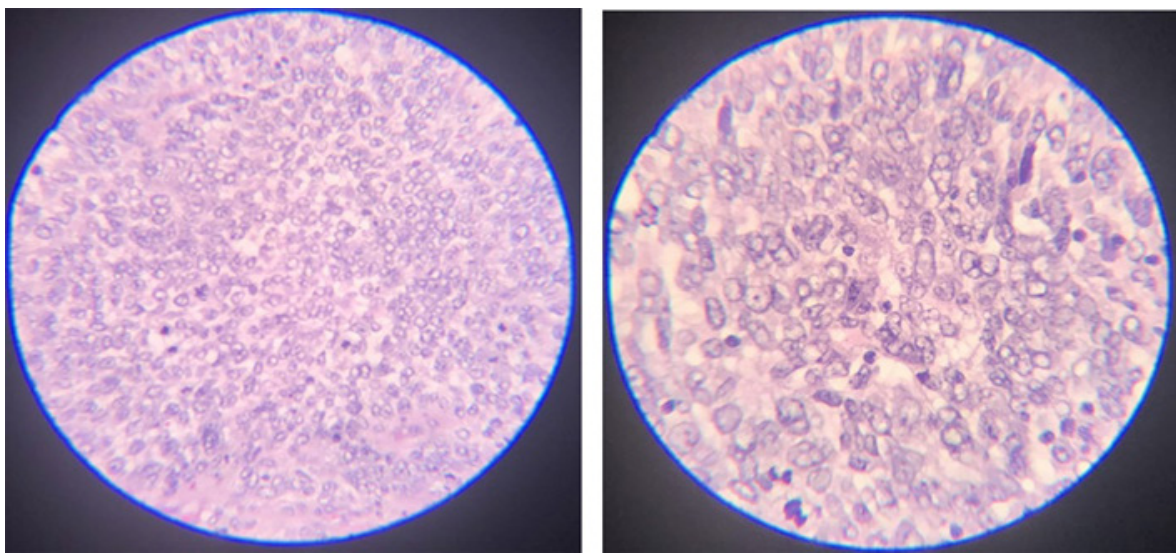

Fig. 2. H\&E staining of breast biopsy.
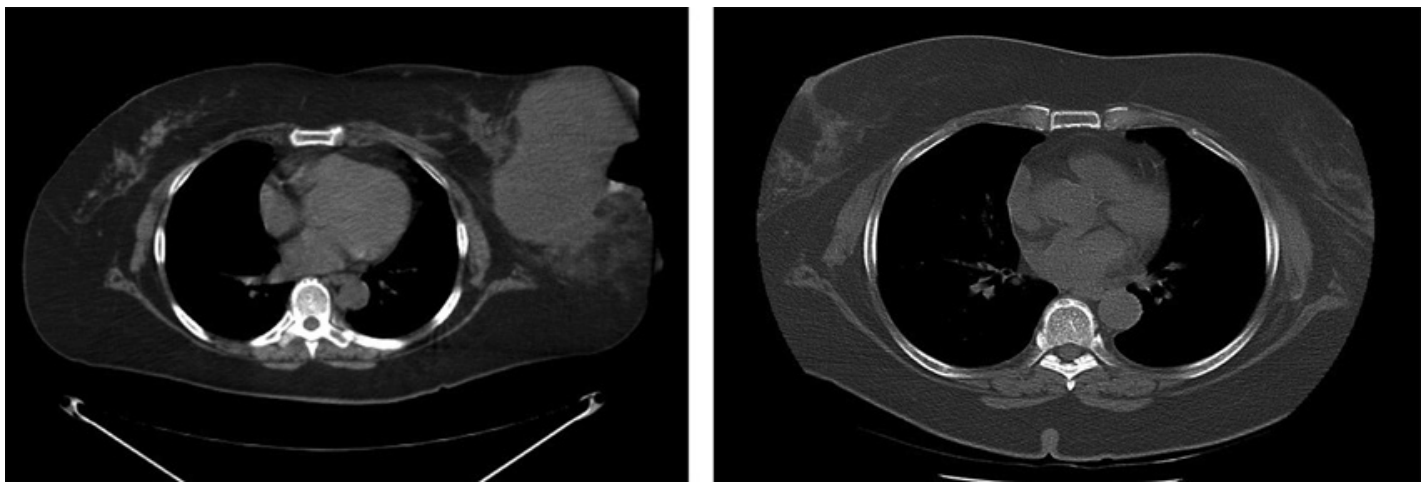

Fig. 3. CT scan before and after treatment with 4 cycles of chemotherapy. 
with left axillary lymphadenopathy $7.6 \times 4.8 \mathrm{~cm}$ with an SUV of 8.34 . She also had right axillary lymphadenopathy, SUV 2.84, with the largest lymph node being $0.5 \mathrm{~cm}$ (Fig. 3). Right axillary lymphadenopathy was determined to be reactive and too small for biopsy. There were no lung lesions and no signs of distant metastasis.

She was classified as stage 3 based on the American Joint Committee on Cancer TNM staging system. Her case was discussed in a multidisciplinary tumor conference, and given the large size of the tumor, localized disease, and axillary lymph node involvement, her treatment plan included 6 cycles of neoadjuvant chemotherapy, followed by surgery, and subsequent radiation treatments. She was initially treated using cisplatin and etoposide chemotherapy, but due to development of hearing difficulty, cisplatin was switched to carboplatin after cycle 3. She had a dramatic reduction in the size of the tumor (Fig. 1, 3) with neoadjuvant chemotherapy. She then underwent a modified radical mastectomy of the left breast with left axillary lymph node dissection. Pathology showed that she had 1 out of 14 lymph nodes that were positive for malignancy. Margins were clear and ductal carcinoma in situ was not seen. At the time of writing this article, she has completed her chemotherapy and surgical treatments and is currently undergoing RT. Given the lack of trials and established guidelines for the use of RT in this disease, clinical judgment was employed based upon the known utility of postmastectomy RT in patients with node-positive invasive ductal carcinoma of the breast, and in the treatment of limited-stage small-cell carcinoma of the lung $[9,10]$. A plan was developed using mixed-energy photons to deliver 5,000 cGy to the chest wall, axilla, internal mammary nodes, and supraclavicular nodes, followed by a 1,000-cGy boost to the scar using electrons. Deep inspiration breath hold technique was used to minimize the dose to the heart and ipsilateral lung. The case was reviewed and approved at radiation oncology chart rounds at a tertiary hospital prior to initiation of therapy. Genomic analysis of her breast tissue has not been done yet due to absence of metastatic disease.

\section{Discussion}

Given the rare occurrence of SCBC, no national or international guidelines exist for treatment of this aggressive malignancy. In terms of chemotherapy, we preferred using neoadjuvant chemotherapy to shrink the tumor given its large size to increase the likelihood of a complete surgical resection and eliminating microscopic tumor cells. In the 56-case review series, 25 patients underwent adjuvant chemotherapy and 15 patients received neoadjuvant chemotherapy. Various chemotherapy regimens have been employed. These include adriamycin-based chemotherapy used in invasive breast tumors, platinum-based chemotherapy regularly employed for SCLC, and FEC (5-fluorouracil, epirubicin, and cyclophosphamide). Thirty patients underwent mastectomy, and 16 patients underwent lumpectomy. $41 \%$ of patients received RT ( $n=23 / 56)$ [8]. Yildirim et al. [11] describe a 6-patient case series and utilized Ki67 in deciding on the chemotherapy regimen for breast neuroendocrine tumors. For tumors with Ki67 $>15 \%$, platinum compounds and etoposide were used, and for Ki67 $<15 \%$, an adriamycin-based regimen was given. Ki67 was $70 \%$ in our patient and she was treated with platinum agents and etoposide.

Wang et al. [12] did a population-based study on invasive neuroendocrine carcinoma of the breast from the SEER (Surveillance, Epidemiology and End Results) database. This study reviewed 142 cases that showed that neuroendocrine carcinoma is an aggressive variant of invasive breast cancer that mostly occurs in women over 60 years of age and usually presents with a tumor size $>2 \mathrm{~cm}$. They also determined that not only was there a stage-for-stage poorer 5-year overall survival rate in neuroendocrine carcinomas of the breast when compared to other invasive mammary carcinomas, but also that age $>60$ years and positive

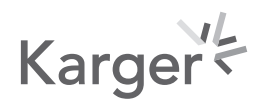


lymph node status were independent prognostic factors for poorer overall survival in neuroendocrine carcinomas.

Estrogen receptor (ER) and progesterone receptor (PR) positivity has been shown to be variable in different case series. Shin et al. [6] reported 67\% ER-positive and 56\% PR-positive cases, Adegbola et al. [5] reported 3 cases, all of them being triple negative, and McCullar et al. [13] reported 31\% ER-positive and 13\% PR-positive cases. Her2/neu receptors are almost always negative. Patients positive for ER or PR have been treated with hormonal agents in accordance with NCCN treatment guidelines for invasive breast cancer. Additionally, the presence of a ductal carcinoma in situ (DCIS) component points to a mammary origin of the tumor [14]. Our patient was triple negative in her receptor status and did not have presence of a DCIS component.

Next generation sequencing of SCBC and SCLC tissue has revealed differences in the genomic landscape of both tumors. PIK3CA mutations were present in 33\% of SCBC cases $(n=19)$ and were absent in all SCLC cases $(n=58, p=0.0005, \mathrm{OR}=0.02)$. TP53 mutations were present in $75 \%$ of cases of SCBC and SCLC. These PIK3CA mutations reportedly occur in approximately $40 \%$ of hormone receptor (ER/PR)-positive and HER2-negative breast cancers [11]. Fortunately, a new immunochemotherapy agent called alpelisib, a PI3K $\alpha$-specific inhibitor, has been developed and investigated. The phase 3 trial that compared treatment with alpelisib-fulvestrant to placebo-fulvestrant showed significantly prolonged progressionfree survival in PIK3CA-mutated, hormone receptor-positive, HER2-negative advanced breast cancer patients who had previously received aromatase inhibitor therapy [15]. Alpelisib is now approved by the FDA (Food and Drug Administration) for this tumor type, and further research is recommended to investigate alpelisib as a potential treatment option in PIK3CAmutated SCBC.

\section{Conclusion}

Even though SCBC is a very rare form of breast cancer, continuing to publish individual case studies will add much needed and valuable information for future studies. For diagnosing primary SCBC, imaging studies are very important to rule out metastasis from SCLC. Data used for SCBC treatment is usually extrapolated from studies done on patients with invasive breast cancer and SCLC. Ki67 can be a tool to help determine the chemotherapy regimen of choice for this rare tumor with no established treatment guidelines. While it might share characteristics with SCLC, it is a unique cancer that needs more research to determine a standard treatment protocol. Additional investigation into PIK3CA mutations may also yield better treatment options in order to improve prognosis for patients diagnosed with SCBC.

\section{Statement of Ethics}

The paper is exempt from ethical committee approval. Written informed consent was obtained from the patient for publication of this case report and any accompanying images. All efforts were made to de-identify the picture and images. The authors have no ethical conflicts to disclose.

\section{Conflict of Interest Statement}

The authors have no conflicts of interest to declare.

\section{Karger'}




\section{Case Reports in Oncology}

Case Rep Oncol 2021;14:761-766

\begin{tabular}{l|c|c|}
\hline DOI: 10.1159/000515505 & @ 2021 The Author(s). Published by S. Karger AG, Basel
\end{tabular} www.karger.com/cro

\section{Funding Sources}

There were no funding sources.

\section{Author Contributions}

A.M. and L.A. conceptualized and prepared the manuscript. T.H. reviewed her biopsy samples and provided pathology pictures. T.M., B.P.Q., and A.M. were involved in clinical care of the patient. L.A., A.M., and B.P.Q. were involved in editing the manuscript. All authors have read and approve the final version of the manuscript.

\section{References}

1 Mirza IA, Shahab N. Small cell carcinoma of the breast. Semin Oncol. 2007;34(1):64-6.

2 Ibrahim NB, Briggs JC, Corbishley CM. Extrapulmonary oat cell carcinoma. Cancer. 1984;54(8):1645-61.

3 Howard S, O’Regan K, Jagannathan J, Krajewski K, Giardino A, Ramaiya N. Extrapulmonary small cell carcinoma: a pictorial review. AJR Am J Roentgenol. 2011;197(3):W392-8.

4 Tan PH, Ellis I, Allison K, Brogi E, Fox SB, Lakhani S, et al. The 2019 World Health Organization classification of tumours of the breast. Histopathology. 2020;77(2):181-5.

5 Adegbola T, Connolly CE, Mortimer G. Small cell neuroendocrine carcinoma of the breast: a report of three cases and review of the literature. J Clin Pathol. 2005;58(7):775-8.

6 Shin SJ, DeLellis RA, Ying L, Rosen PP. Small cell carcinoma of the breast: a clinicopathologic and immunohistochemical study of nine patients. Am J Surg Pathol. 2000;24(9):1231-8.

7 Kanat O, Kilickap S, Korkmaz T, Ustaalioglu Oven BB, Canhoroz M, Cubukcu E, etal. Primary small cell carcinoma of the breast: Report of seven cases and review of the literature. Tumori. 2011;97(4):473-8.

8 Boutrid H, Kassem M, Tozbikian G, Morgan E, White J, Shah M, et al. TTF-1 Positive Primary Small Cell Carcinoma of the Breast: A Case Report and Review of the Literature. Front Endocrinol (Lausanne). 2020;11: 228.

9 Overgaard M, Jensen MB, Overgaard J, Hansen PS, Rose C, Andersson M, et al. Postoperative radiotherapy in high-risk postmenopausal breast-cancer patients given adjuvant tamoxifen: Danish Breast Cancer Cooperative Group DBCG 82c Randomised trial. Lancet. 1999 May 15;353(9165):1641-8.

10 Overgaard 1 M, Hansen PS, Overgaard J, Rose C, Andersson M, Bach F, et al. Postoperative radiotherapy in highrisk premenopausal women with breast cancer who receive adjuvant chemotherapy. Danish Breast Cancer Cooperative Group 82b Trial. N Engl J Med. 1997 Oct;337(14):949-55.

11 Yildirim Y, Elagoz S, Koyuncu A, Aydin C, Karadayi K. Management of neuroendocrine carcinomas of the breast: A rare entity. Oncol Lett. 2011 Sep 1;2(5):887-90.

12 Wang J, Wei B, Albarracin CT, Hu J, Abraham SC, Wu Y. Invasive neuroendocrine carcinoma of the breast: a population-based study from the surveillance, epidemiology, and end results (SEER) database. BMC Cancer. 2014 Mar; 14:147.

13 McCullar B, Pandey M, Yaghmour G, Hare F, Patel K, Stein M, et al. Genomic landscape of small cell carcinoma of the breast contrasted to small cell carcinoma of the lung. Breast Cancer Res Treat. 2016;158(1):195-202.

14 Inno A, Bogina G, Turazza M, Bortesi L, Duranti S, Massocco A, et al. Neuroendocrine carcinoma of the breast: current evidence and future perspectives. Oncologist. 2016;21(1):28-32.

15 André F, Ciruelos E, Rubovszky G, Campone M, Loibl S, Rugo HS, et al. Alpelisib for PIK3CA-Mutated, Hormone Receptor-Positive Advanced Breast Cancer. N Engl J Med. 2019;380(20):1929-40. 